\title{
Spontaneous formation of nonspherical water ice grains in a plasma environment
}

\author{
Kil-Byoung Chai ${ }^{1}$ and Paul M. Bellan ${ }^{1}$
}

Received 8 October 2013; revised 4 November 2013; accepted 6 November 2013; published 3 December 2013.

[1] Saturn's rings, terrestrial polar mesospheric clouds, and astrophysical molecular clouds are all dusty plasma environments where tiny grains of water ice are an important constituent. Existing models typically assume that the ice grains are spherical and then invoke various arguments about the normal distribution or the power law dependence of grain number density on grain radius. Using a laboratory plasma in which water ice grains spontaneously form, we investigated the validity of the traditional assumption that these grains are spherical. We found that in certain cases at low ambient pressures, water ice grains in the laboratory dusty plasma are not spherical but instead are highly elongated, i.e., ellipsoidal. Preliminary analysis suggests that electrical forces associated with the dusty plasma environment are responsible for the nonspherical shape. Citation: Chai, K.-B., and P. M. Bellan (2013), Spontaneous formation of nonspherical water ice grains in a plasma environment, Geophys. Res. Lett., 40, 6258-6263, doi:10.1002/2013GL058268.

\section{Introduction}

[2] Plasmas containing water ice grains are ubiquitous. Examples include certain of Saturn's rings [Goertz, 1989], terrestrial polar mesospheric clouds [Havnes et al., 1996], astrophysical molecular clouds [McClure et al., 2012], and comet tails [Davies et al., 1997]. Water ice grains in Saturn's B ring are responsible for spoke-like patterns [Goertz and Morfill, 1983; Mitchell et al., 2006]; water ice grains in Saturn's F ring, influenced by the gravitational field of nearby small satellites, make wave-like structures [Murray et al., 2008]; water ice grains ejected from the south pole of Enceladus [Porco et al., 2006] populate Saturn's diffuse E ring. On Earth, water ice grains in polar mesospheric clouds cause strong radar echoes known as polar summertime mesospheric echoes [Rapp and Lubken, 2003]; these radar echoes result from the water ice modulating the spatial distribution of electrons to produce a Bragg reflection. Water ice grains in polar mesospheric clouds in addition scavenge and deplete the meteoric iron layer [Plane et al., 2004]. Water ice grains in astrophysical molecular clouds can cool the cloud, determine cloud opacity, store oxygen atoms, and enhance the agglomeration of planetesimals [McClure et al., 2012].

[3] Previous studies of these plasmas typically assumed the water ice grains to be spherical. Some of these studies

\footnotetext{
${ }^{1}$ Department of Applied Physics and Materials Science, California Institute of Technology, Pasadena, California, USA

Corresponding author: K.-B. Chai, Department of Applied Physics and Materials Science, California Institute of Technology, 1200 E. California Blvd., Pasadena, CA 91125, USA. (kbchai@caltech.edu)

C2013. American Geophysical Union. All Rights Reserved. 0094-8276/13/10.1002/2013GL058268
}

assumed that the spherical grains are monodisperse or that the number density of the spherical grains has a normal distribution [Goertz, 1989; Havnes et al., 1996; Rapp and Lubken, 2003; Plane et al., 2004; Mitchell et al., 2006] while others assumed that the number density has a power law dependence on radius of the form $n \sim r^{-q}$ where $q$ is adjusted for best fit to observations or to provide inputs for theories [Zubko et al., 2004; Horanyi et al., 2008; Kempf et al., 2008; McClure et al., 2012]. However, this long-standing conventional assumption of spherical shape seems to be for mathematical convenience rather than from actual measurement. Furthermore, it has been proposed both for Saturn's rings [Dollfus, 1996] and for mesospheric clouds [Gadsden et al., 1979; Rapp et al., 2007] that observed light scattering by ice particles might be better explained if the ice grains were assumed to be nonspherical, e.g., needle shaped.

[4] There are two good reasons to believe that in a plasma environment nonspherical, elongated growth of water ice should be more likely than spherical growth. The first reason is that nonspherical ice has often been seen in nonplasma environments. Bartlett et al. [1963] and Libbrecht and Tanusheva [1998] investigated the effect of imposed electric potentials on water ice attached to a substrate in atmospheric pressure, nonplasma settings. They found that when thus electrified, water ice develops fast-growing needle shapes. Nonspherical shapes have also been seen at high pressures in unelectrified, nonplasma settings [Bailey and Hallett, 2004]. The electrical charging of water ice grains in a plasma environment leads to the second reason. Since water molecules have a large dipole moment and so are attracted to the gradient of an electric field and since large electric field gradients occur at regions of small radius of curvature, water molecules incident upon a charged ellipsoidal ice grain will be preferentially attracted to the ends of the ellipsoid much like iron filings are attracted to the ends of a bar magnet. This preferential accretion to the ellipsoid ends will accentuate any initial ellipticity.

[5] In order to investigate whether or not water ice grains in a plasma are spherical, we built a laboratory experiment similar to that reported by Shimizu et al. [2010] in which water ice grains spontaneously develop and then, unlike Shimizu et al., determined both the size and shape of these grains. We found that under certain conditions the spontaneously generated water ice grains are not spherical but instead are highly elongated, i.e., are ellipsoidal.

\section{Relevance to Natural Plasmas}

[6] The water ice dusty plasma experiment has parameters relevant to naturally occurring water ice dusty plasmas and so the experimental results have implications for a wide range of situations. Table 1 shows plasma and dust grain parameters 
Table 1. Comparison Between Two Regimes for the Caltech Experiment and Some Representative Parameters for Various Ice Dusty Plasmas Occurring in Nature

\begin{tabular}{|c|c|c|c|c|c|c|c|c|}
\hline & $\begin{array}{l}\text { Neutral } \\
\text { Density }\end{array}$ & $\begin{array}{l}\text { Plasma } \\
\text { Density }\end{array}$ & $\begin{array}{c}\text { Ion } \\
\text { Temperature }\end{array}$ & $\begin{array}{l}\text { Electron } \\
\text { Temperature }\end{array}$ & $\begin{array}{l}\text { Grain } \\
\text { Radius }\end{array}$ & $\begin{array}{l}\text { Grain } \\
\text { Density }\end{array}$ & $\begin{array}{l}\text { Havnes } \\
\text { Parameter }\end{array}$ & References \\
\hline & $\left(\mathrm{cm}^{-3}\right)$ & $\left(\mathrm{cm}^{-3}\right)$ & $(\mathrm{eV})$ & $(\mathrm{eV})$ & $(\mu \mathrm{m})$ & $\left(\mathrm{cm}^{-3}\right)$ & & \\
\hline Caltech Experiment & $10^{16}$ & $10^{8}$ & 0.01 & 1 & $\begin{array}{l}0.1 \\
10\end{array}$ & $\begin{array}{l}10^{7} \\
10^{4}\end{array}$ & $\begin{array}{c}7 \\
7 \times 10^{-1}\end{array}$ & \\
\hline Polar Mesospheric Cloud & $10^{14}$ & 4000 & 0.01 & 0.01 & $10^{-3}-10^{-1}$ & $0-1000$ & $5 \times 10^{-1}$ & Rapp and Lubken [2004] ${ }^{\mathrm{a}}$ \\
\hline Saturn's AB Ring & 1000 & 40 & 5 & 5 & 1 & $10^{-2}$ & $9 \times 10^{-1}$ & Yaroshenko et al, [2007] \\
\hline Saturn's G Ring & 1000 & 10 & 10 & 1 & 1 & $10^{-7}$ & $7 \times 10^{-6}$ & $\begin{array}{l}\text { Frank et al. }[1980], \\
\text { Goertz [1989], Ip [2000], } \\
\text { and Cravens et al. }\end{array}$ \\
\hline Saturn's E Ring & $10^{2}-10^{4}$ & 10 & 30 & 5 & 1 & $10^{-7}$ & $3 \times 10^{-5}$ & $\begin{array}{l}\text { Frank et al. }[1980], \text { Goertz [1989], } \\
\text { Ip [2000]; Cravens et al. }[2009]\end{array}$ \\
\hline Molecular Cloud & $10^{6}$ & 1 & 0.001 & 1 & 0.1 & $<10^{-3}$ & $<7 \times 10^{-2}$ & Shu et al. [1987], Zubko et al. [2004] \\
\hline
\end{tabular}

${ }^{\mathrm{a}}$ Grain density was assumed to be log-normal function of grain radius when calculating Havnes parameter.

${ }^{\mathrm{b}}$ These parameters are quite nominal and, in general, all these plasmas have substantial ranges of parameters.

for our plasma and several naturally occurring ice dusty plasmas as well as the Havnes dimensionless parameter $P=4 \pi r_{d} n_{d} \lambda_{D e}^{2}$ [Havnes et al., 1987] that characterizes the ice dusty plasma regime. Plasma parameters of our experiment are assumed values based on similar RF plasmas while grain parameters are measured values. Parameters of other applications are from references listed in the right-most column. If $P>10$, the majority of available electrons are captured by a large number of small dust grains so few free electrons remain in the plasma; if $P<0.1$ there is a small number of large, highly charged dust grains but most electrons are free and reside in the plasma. Because of the wide range of experimental regimes, it is expected that results from the experiment should be relevant to a wide range of natural plasmas.

\section{Caltech Ice Dusty Plasma Apparatus}

[7] Figure 1 is a schematic sketch of our apparatus which differs from that used by Shimizu et al. [2010] by having direct injection of water vapor rather than in situ creation from $\mathrm{D}_{2}$ and $\mathrm{O}_{2}$ and also by having an adjustable gap between electrodes. The main chamber is a $15.24 \mathrm{~cm}$ spherical square stainless steel vacuum chamber. The electrically and thermally insulated electrodes are two $6 \mathrm{~cm}$ diameter parallel aluminum disks separated by a $1.4 \mathrm{~cm}$ gap. These electrodes are cooled by cold fingers and are electrically connected to the RF generator. Each electrode is mounted on a stainless steel bellows that enables adjustment of the interelectrode gap. The electrodes are powered by a custom-made $13.56 \mathrm{MHz}$ radio frequency (RF) generator that applies 1-3 watts of RF to ignite and sustain the plasma. Argon (Ar) gas from a compressed gas cylinder and water vapor from a small sealed stainless tank containing distilled water are delivered to the main chamber through two respective variable leak valves. The chamber pressure is monitored using an ion gauge and a capacitance manometer.

[8] The experimental procedure is as follows: Residual gas is pumped out of the distilled water tank for $2 \mathrm{~h}$ by switching valves to connect the vacuum pumps to the water tank. After this purging process, liquid nitrogen is poured into the upper and lower liquid nitrogen containers and the electrodes are allowed to cool for $45 \mathrm{~min}$. The main vacuum chamber is then filled with 100-600 mTorr (13 Pa to 80 Pa) Ar gas with the pressure being measured by the capacitance manometer. The plasma is ignited with $1-3 \mathrm{~W}$ RF power. After plasma

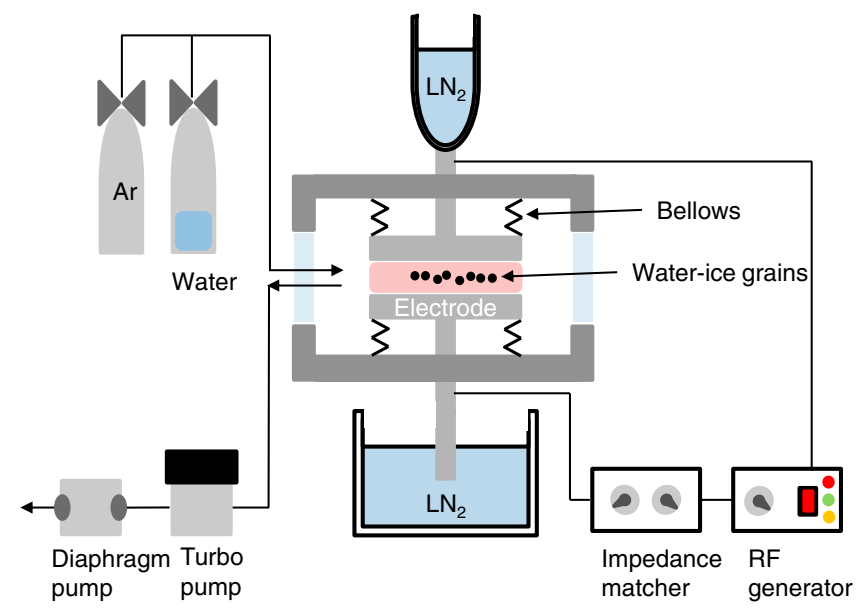

Figure 1. Schematic sketch of Caltech ice dusty plasma apparatus. Our apparatus is an extension of that reported by Shimizu et al. [2010] but differs in two important ways. First, we use direct injection of water vapor into a preexisting background Ar plasma rather than in situ creation of heavy water vapor in a deuterium-oxygen plasma and second, we have an adjustable interelectrode gap. Direct injection of water vapor enables precise control of the amount of water vapor in the system. 

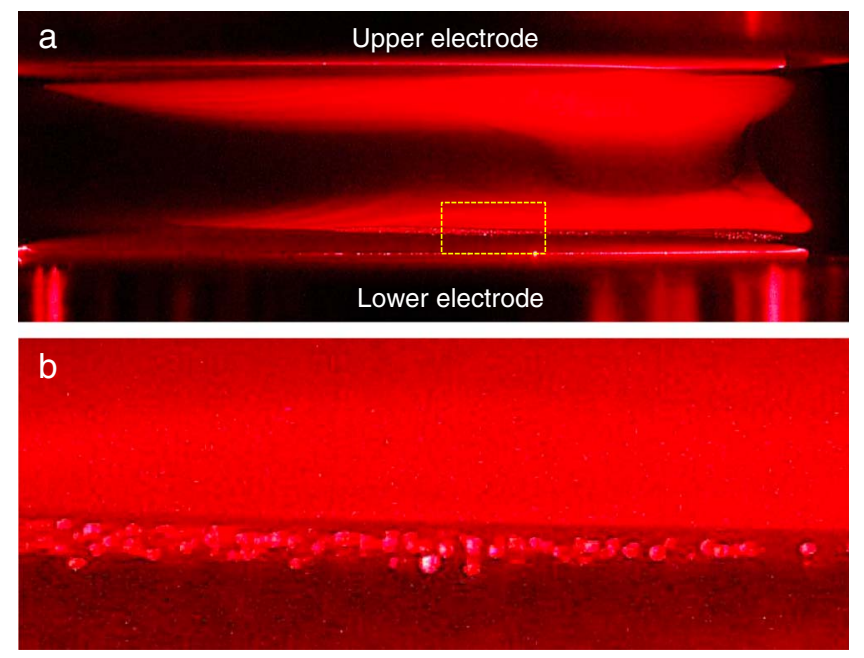

Figure 2. (a) Cloud of water ice grains generated in the laboratory plasma. The ice grains are observed by illuminating the volume with a vertical sheet of He-Ne laser light $(632.8 \mathrm{~nm}, 5 \mathrm{~mW})$ and photographing at right angles to the laser propagation direction. (b) 7 times magnified image of the white rectangular region in Figure 2a shows larger, discrete ice grains levitating near the bottom electrode.

ignition, $5 \mathrm{mTorr}(0.6 \mathrm{~Pa})$ water vapor is introduced using the water system variable leak valve. The water vapor pressure is measured by noting the increase in capacitance manometer reading. Immediately upon introduction of the water vapor, water ice grains are observed to form. It is observed that water ice grains are only generated when there is plasma; this requirement for plasma was also observed by Shimizu et al. [2010].

\section{Results and Discussions}

[9] Water ice grains spontaneously formed in the plasma are shown in Figures $2 \mathrm{a}$ and $2 \mathrm{~b}$. Most grains levitate near the upper or lower electrode sheaths (upper and lower clouds in Figure 2a) and, as seen in Figure 2a, are so small that they cannot be individually distinguished. However, as seen in Figure 2b, which is a 7 times magnified image of the white rectangle region in Figure 2a, grains levitating near the bottom electrode are sufficiently large to be individually distinguished.

[10] Laser diffraction was used to determine both the size and shape of the large grains levitating near the bottom electrode. This determination exploits Babinet's principle, wherein each ice-dust grain produces a Fraunhofer diffraction pattern equivalent to a pinhole having the same dimensions. In fact, the diffraction pattern is effectively the 2-D Fourier transform of the projected ice-dust grain shape so ellipticity of the diffraction pattern provides information about ellipticity of the dust grains. Furthermore, the diffraction patterns of many grains coherently superimpose if the dust grains illuminated by the laser beam are monodisperse and approximately equi-distant from the screen on which the diffraction pattern is projected.

[11] Figures 3a-3d show that significant changes in diffraction pattern occur when the Ar background pressure is
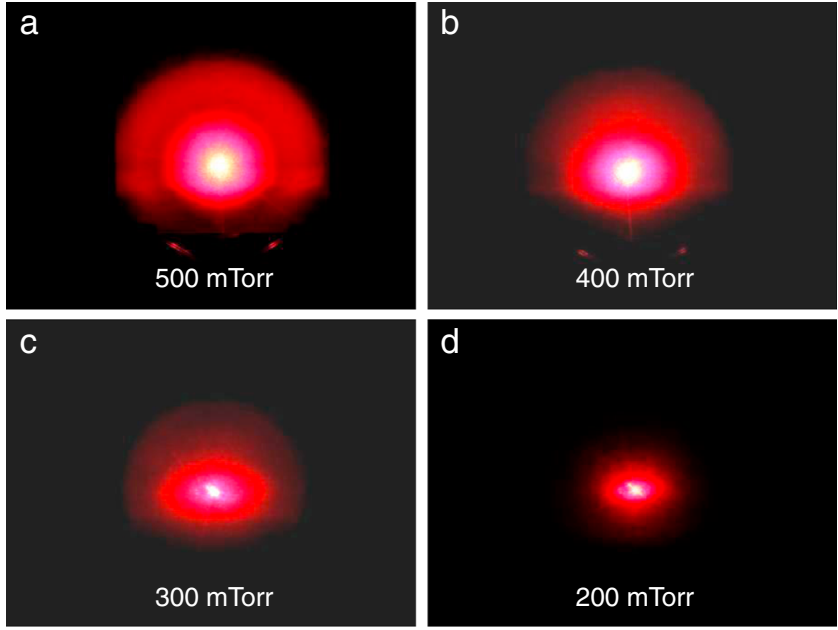

Figure 3. (a)-(d) Laser diffraction images of ice grains for various Ar background pressures. The water vapor pressure and RF power were fixed. The laser beam traversed the region where large grains are levitating near the bottom electrode and the resulting diffraction pattern was displayed on a ground glass screen and photographed. The lower part of the diffraction pattern is obstructed by the bottom electrode. 

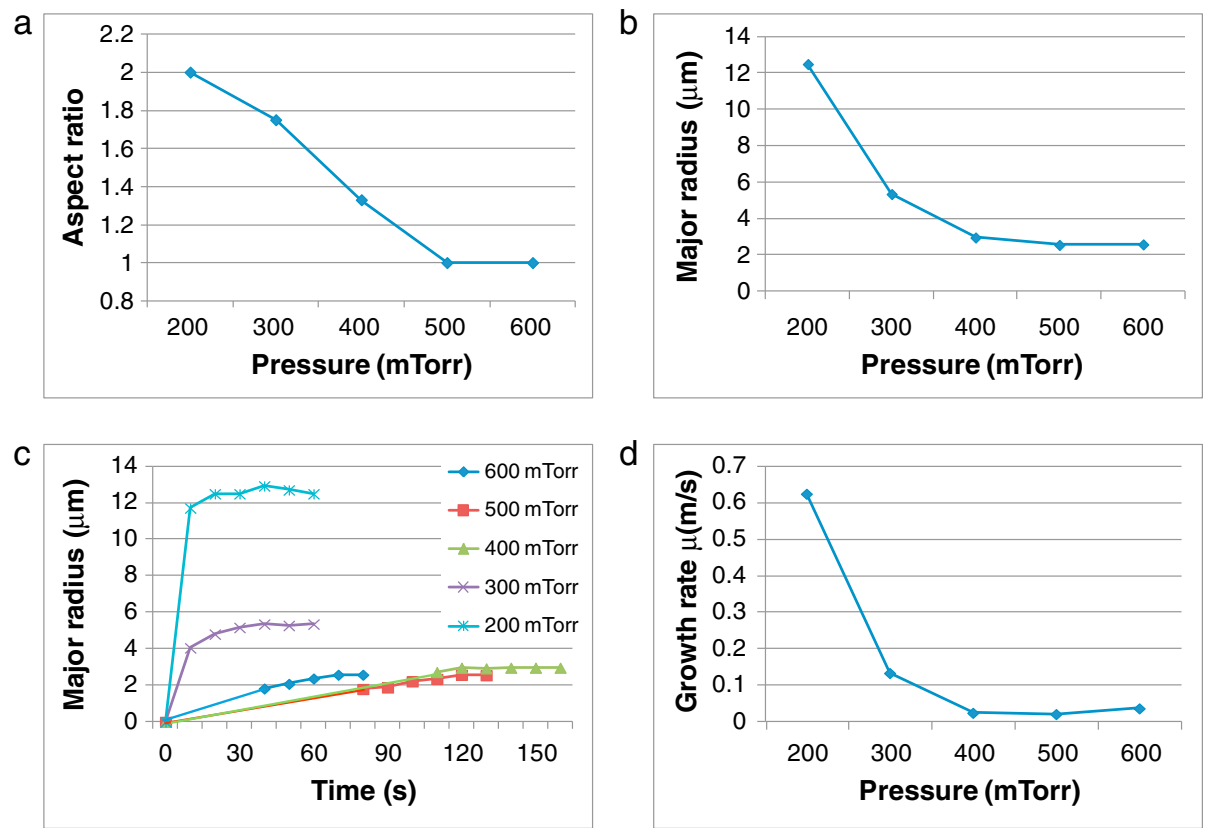

Figure 4. (a) Aspect ratio, defined as the ratio of major to minor radius, increases as the background pressure is decreased. The diffraction pattern at 100 mTorr, was not as clear as at higher pressures perhaps because of polydispersity in grain size and shape or because being small, was difficult to resolve. (b) Major radius of maximum size ice grains as a function of Ar background pressure. This radius was measured from the size of first minimum of the minor radius of the diffraction pattern after calibration with a known-sized pinhole. (c) Time evolution of major radius of ice grains for a range of background pressures. In all cases, the ice grains grow fast initially but then growth stops. (d) Dependence of average growth rate on Ar background pressure. This dependence shows that the average growth rate is inversely proportional to background pressure suggesting that collisions of water molecules with Ar background gas inhibit ice grain growth.

varied. The diffraction pattern is large and circular at 500 mTorr implying that the ice grains are small and spherical at this pressure. However, the diffraction pattern becomes small and horizontally elongated as the Ar pressure is decreased indicating that at low Ar pressures, the ice grains are monodisperse, large, and ellipsoidal with vertical orientation. The aspect ratio, defined as the ratio of diffraction pattern major radius to minor radius, changes from 1.0 to 2.0 as the background pressure is decreased from 600 mTorr to 200 mTorr (see Figure 4a). As will be shown later, direct imaging reveals that the aspect ratio reaches 2.5 at 100 mTorr.

[12] The diffraction pattern is observed to become smaller with time implying temporal growth of ice grain size. The

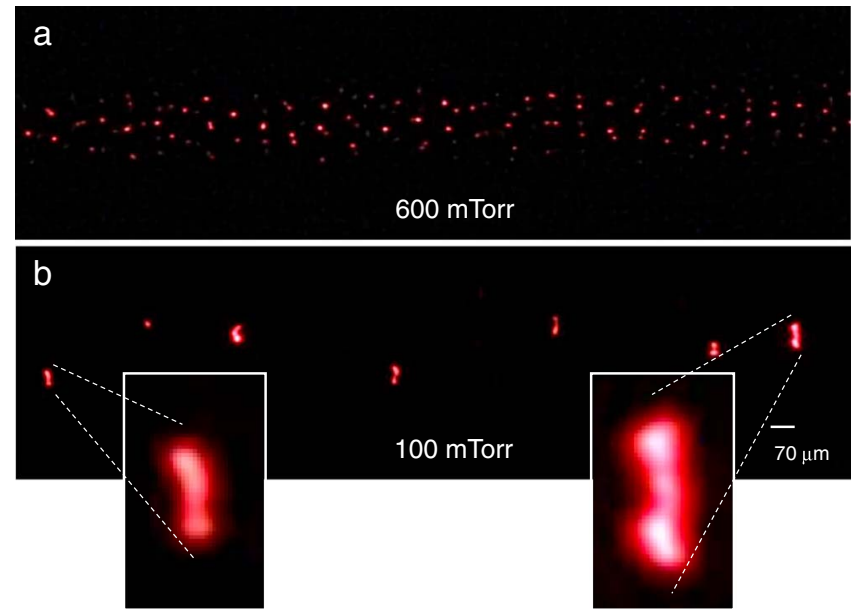

Figure 5. Direct images of water ice grains obtained at (a) 600 mTorr and (b) 100 mTorr Ar background pressures, respectively. The photos were taken with a 2 times magnification telescopic lens and Canon DSLR camera with $4.7 \mu \mathrm{m}$ by $4.3 \mu \mathrm{m}$ pixel size so each pixel in the image corresponds to approximately $2 \mu \mathrm{m}$ by $2 \mu \mathrm{m}$. A short exposure time ( $1 / 4000 \mathrm{~s})$ was used to avoid recording a blurred trajectory of a moving grain (average velocity is a few mm/s). A maximum entropy deconvolution was performed to improve the camera resolution. It is clearly seen that as the background pressure is decreased, ice grains become large and vertically elongated and also that the number density of the ice grains decreases. 
diffraction patterns in Figures 3a-3d were obtained when the diffraction pattern became steady-state implying that grain growth had ceased. The dependence of saturated maximum radius on Ar background pressure is shown in Figure 4b. This shows that ice grains can grow to $12 \mu \mathrm{m}$ major radius at $200 \mathrm{mT}$ orr background pressure but only to $2.5 \mu \mathrm{m}$ radius at 600 mTorr.

[13] Figure 4c, a plot of the time evolution of ice grain major radius for a range of Ar background pressures, shows that ice grains have a rapid initial growth but after a while growth stops and the size becomes constant. This saturation of the growth suggests that the density of free water molecules becomes depleted as the ice grains grow. An average ice grain growth rate, defined as the maximum major radius divided by the time to reach this maximum major radius, was calculated for each Ar pressure. These average growth rates, shown in Figure 4d, show that fastest growth occurs at lowest Ar background pressure. This observed inverse dependence of growth on background pressure indicates that collisions of water molecules with Ar background gas molecules inhibit ice grain growth and so contradicts the generally used ice particle growth model of Hesstvedt [1961] which predicts that grain growth rate depends only on water vapor partial pressure and not on the nonwater background pressure.

[14] The large ice grain size at low Ar background pressure suggests that direct imaging should be possible. Direct imaging proved to be successful and confirmed the implications of the diffraction patterns. Direct images of ice grains at 600 mTorr and 100 mTorr Ar background pressures are shown in Figures 5a and 5b. At 600 mTorr the average grain size is small (about $6 \mu \mathrm{m}$ in diameter) while at $100 \mathrm{mTorr}$ the grains are large and vertically elongated (50 $\mu \mathrm{m}$ length, 20 $\mu \mathrm{m}$ width). In addition, the ice grain number density $n_{\mathrm{d}}$ decreases from $10^{5} \mathrm{~cm}^{-3}$ to $10^{4} \mathrm{~cm}^{-3}$ as the background pressure decreases. The observed aspect ratio of 2.5 at 100 mTorr is consistent with the trend in aspect ratios from the diffraction measurements (see Figure 4a).

[15] We conjecture that the critical background pressure for onset of aspherical ice grain growth is determined by competition between the mean free path of water molecules (inversely proportional to Ar background pressure) and the effective Debye shielding length of charged ice grains. This effective Debye shielding length is of the order of the ion Debye length in a dusty plasma. The explanation that incident water molecules accrete to the ends of a charged ellipsoidal ice grain requires that the incident water molecules have collisionless orbital motion and experience the electric field of the charged ice grain over some distance.

[16] When the mean free path exceeds the effective Debye length, the water molecules incident on a charged ice grain experience the detailed geometrical morphology of the ice grain electric field during their collisionless orbital motion and so can target regions of high electric field on the dust grain [Stark et al., 2006]. On the other hand, when the molecular mean free path is shorter than the effective Debye length, collisions between water molecules and background gas continuously scatter water molecules so they cannot target any particular portion of the ice grain, leading to a uniform, spherical grain growth. At a nominal assumed ion density of $10^{8} \mathrm{~cm}^{-3}$ and a $400 \mathrm{mTorr}$ Ar background pressure the mean free path of a water molecule is $80 \mu \mathrm{m}$ while the ion Debye length is $84 \mu \mathrm{m}$ so the crossover from collisional behavior to orbital motion collisionless behavior should occur within the range of Ar pressures being used.

\section{Conclusion}

[17] Our results demonstrate that ice grains spontaneously form in a suitably cooled plasma containing water vapor. No external nucleation material is required, i.e., homogeneous ionic nucleation takes place. Furthermore, the ice grains are aspherical when the background pressure is lower than a certain pressure and presumably this aspherical shape develops soon after nucleation rather than later when the grains are large. This result implies that it is plausible that aspherical ice grains are common in naturally occurring plasmas (Saturn's rings, polar mesosphere clouds, and molecular clouds) in which case existing models assuming spherical grains would have to be revised. This is important because ellipsoidal grains will have significantly different interactions with electromagnetic radiation in terms of effective cross section and in terms of how scattered light depends on the polarization of incident light [Holland and Gagne, 1970]. In particular, just as long chaff scatters radar most efficiently, highly elongated grains will provide much stronger scattering of electromagnetic radiation than the same mass arranged in a sphere. Also, the aerodynamic drag properties of ellipsoidal grains will be quite different from spherical grains [Gadsden et al., 1979].

[18] Our future plans include determining what limits ice grain size, obtaining more highly resolved ice grain images, and finding what causes the vertical alignment of ellipsoidal grains. While this paper has focused on the properties of an individual ice dust grain, we have also seen evidence of strong coupling between ice grains. This shows up in the form of a large-scale dust grain vortex composed of well-defined streamlines separated by small-scale, highly periodic gaps. We plan to investigate whether strong coupling between charged dust grains is responsible for these gaps and to see if these periodically separated streamlines are moving versions of the vertical chains seen in previous dusty plasma experiments [Aschinger and Winter, 2012]. The possibility of forming an ice dusty plasma by non-RF means (e.g., DC electric fields or UV light) will also be investigated as well as the possibility of comparing homogenous to heterogenous nucleation by injection of nanoparticles that serve as nucleation centers for heterogeneous nucleation.

[19] Acknowledgments. This work was supported by the USDOE/ NSF Partnership in Plasma Science. We thank S. Shimizu for graciously providing detailed information on the experimental setup used in Shimizu et al. [2010].

[20] The Editor thanks an anonymous reviewer and Mihaly Horanyi for their assistance evaluating this manuscript.

\section{References}

Aschinger, A., and J. Winter (2012), Dynamics light scattering study of phase transitions in three-dimensional complex plasmas, New J. Phys., 14, 093036, doi:10.1088/1367-2630/14/9/093036.

Bailey, M., and J. Hallett (2004), Growth rates and habits of ice crystals between $-20^{\circ}$ and $-70^{\circ} \mathrm{C}$, J. Atmos. Sci., 61, 514-543, doi:10.1175/15200469(2004)061<0514:GRAHOI $>2.0 . \mathrm{CO} ; 2$.

Bartlett, J. T., A. P. Heuval, and B. J. Mason (1963), The growth of ice crystals in an electric field, Z. Angew. Math. Phys., 14, 599-610, doi:10.1007/ BF01601267.

Cravens, T. E., R. L. McNutt Jr., J. H. Waite Jr., I. P. Robertson, J. G. Luhmann, W. Kasprzak, and W.-H. Ip (2009), Plume ionosphere of Enceladus as seen by the Cassini ion and neutral mass spectrometer, Geophys. Res. Lett., 36, L08106, doi:10.1029/2009GL037811. 


\section{CHAI AND BELLAN: FIRST EVIDENCE OF NONSPHERICAL GROWTH}

Davies, J. K., T. L. Roush, D. P. Cruikshank, M. J. Bartholomew, T. R. Geballe, T. Owen, and C. de Bergh (1997), The detection of water ice in comet Hale-Bopp, Icarus, 127, 238-245, doi:10.1006/icar.1996.5673.

Dollfus, A. (1996), Saturn's rings: Optical reflectance polarimetry, Icarus, 124, 237-261, doi:10.1006/icar.1996.0201.

Frank, L. A., B. G. Burek, K. L. Ackerson, J. H. Wolfe, and J. D. Mihalov (1980), Plasmas in Saturn's magnetosphere, J. Geophys. Res., 85, 5695-5708.

Gadsden, M., P. Rothwell, and M. J. Taylor (1979), Detection of circulary polarised light from noctilucent clouds, Nature, 278, 628-629, doi:10.1038/ $278628 \mathrm{a} 0$.

Goertz, C. K. (1989), Dusty plasmas in the solar system, Rev. Geophys., 27, 271-292.

Goertz, C. K., and G. E. Morfill (1983), A model for the formation of spokes in Saturn's ring, Icarus, 53, 219-229, doi:10.1016/0019-1035(83)90143-4.

Havnes, O., C. K. Goertz, G. E. Morfill, E. Grun, and W. Ip (1987), Dust charges, cloud potential, and instabilities in a dust cloud embedded in a plasma, J. Geophys. Res., 92, 2281-2287.

Havnes, O., J. Trøim, T. Blix, W. Mortensen, L. I. Næsheim, E. Thrane, and T. Tønnesen (1996), First detection of charged dust particles in the Earth's mesosphere, J. Geophys. Res., 101, 10,839-10,847.

Hesstvedt, E. (1961), Note on the nature of noctilucent clouds, J. Geophys. Res., 66, 1985-1987.

Holland, A. C., and G. Gagne (1970), The scattering of polarized light by polydisperse system of irregular particles, Appl. Opt., 9, 1113-1121, doi:10.1364/AO.9.001113.

Horanyi, M., A. Juhasz, and G. E. Morfill (2008), Large-scale structure of Saturn's E-ring, Geophys. Res. Lett., 35, L04203, doi:10.1029/ 2007GL032726.

Ip, W.-H. (2000), Thermal plasma composition in Saturn's magnetosphere Planet. Space Sci., 48, 775-783, doi:10.1016/S0032-0633(00)00036-2.

Kempf, S., U. Beckmann, G. Moragas-Klostermeyer, F. Postberg, R. Srama T. Economou, J. Schmidt, F. Spahn, and E. Grün (2008), The E ring in the vicinity of Enceladus I. Spatial distribution and properties of the ring particles, Icarus, 193, 420-437, doi:10.1016/j.icarus.2007.06.027.

Libbrecht, K. G., and V. M. Tanusheva (1998), Electrically induced morphological instabilities in free dendrite growth, Phys. Rev. Lett., 81, 176-179, doi:10.1103/PhysRevLett.81.176.
McClure, M. K., P. Manoj, N. Calvet, L. Adame, C. Espaillat, D. M. Watson B. Sargent, W. J. Forrest, and P. D'Alessio (2012), Probing dynamical processes in the planet-forming region with dust mineralogy, Astrophys. J., 759, L10, doi:10.1088/2041-8205/759/1/L10.

Mitchell, C. J., M. Horanyi, O. Havnes, and C. C. Porco (2006), Saturn's spokes: Lost and found, Science, 311, 1587-1589, doi:10.1126/science.1123783.

Murray, C. D., K. Beurle, N. J. Cooper, M. W. Evans, G. A. Williams, and S. Charnoz (2008), The determination of the structure of Saturn's F ring by nearby moonlets, Nature, 453, 739-744, doi:10.1038/nature06999.

Plane, J. M. C., B. J. Murray, X. Chu, and C. S. Gardner (2004), Removal of meteoric iron on polar mesospheric clouds, Science, 304, 426-428, doi:10.1126/science. 1093236

Porco, C. C., et al. (2006), Cassini observes the active south pole of Enceladus, Science, 311, 1393-1401, doi:10.1126/science.1123013.

Rapp, M., and F.-J. Lubken (2003), On the nature of PMSE: Electron diffusion in the vicinity of charged particles revisited, J. Geophys. Res., 108(D8), 8437, doi:10.1029/2002JD002857.

Rapp, M., and F.-J. Lubken (2004), Polar mesosphere summer echoes (PMSE): Review of observations and current understanding, Atmos. Chem. Phys., 4, 2601-2633, doi:10.5194/acp-4-2601-2004.

Rapp, M., G. E. Thomas, and G. Baumgarten (2007), Spectral properties of mesospheric ice clouds: Evidence for nonspherical particles, J. Geophys. Res., 112, D03211, doi:10.1029/2006JD007322.

Shimizu, S., B. Klumov, T. Shimizu, H. Rothermel, O. Havnes, H. M. Thomas, and G. E. Morfill (2010), Synthesis of water ice particles in a plasma chamber, J. Geophys. Res., 115, D18205, doi:10.1029/2009JD013375.

Shu, F. H., F. C. Adams, and S. Lizano (1987), Star formation in molecular clouds: Observation and theory, Annu. Rev. Astron. Astrophys., 25, 23-81, doi:10.1146/annurev.aa.25.090187.000323.

Stark, C. R., H. E. Potts, and D. A. Diver (2006), Elliptical dust growth in astrophysical plasmas, Astron. Astrophys., 457, 365-370, doi:10.1051/ 0004-6361:20064922.

Yaroshenko, V. V., F. Verheest, and G. E. Morfill (2007), Dust-acoustic waves in collisional dusty plasmas of planetary rings, Astron. Astrophys., 461, 385-391, doi:10.1051/0004-6361:20066102.

Zubko, V., E. Dwek, and R. G. Arendt (2004), Interstellar dust models consistent with extinction, emission, and abundance constraints, Astrophys. J. Suppl. Ser., 152, 211-249, doi:10.1086/382351. 